\title{
Aprendizaje de y con robótica, algunas experiencias ${ }^{1}$ \\ Learning of and with Robotics, some experiences
}

\author{
Pedro Antonio López Ramírez $z^{2}$ \\ Estudiante de Maestría en Ingeniería de Sistemas e Informática \\ Universidad Industrial de Santander \\ Bucaramanga, Colombia \\ palopez@uis.edu.co
}

Hugo Andrade Sosa ${ }^{3}$

Profesor titular

Universidad Industrial de Santander

Director Grupo de Investigación SIMON

Bucaramanga, Colombia

handrade@uis.edu.co

Recibido: 6 junio 2012 Corregido: 14 abril 2013 Aceptado: 16 junio 2013

1 Este artículo se presenta en nombre de la Universidad Industrial de Santander (UIS), por integrantes del grupo SIMON de Investigación en Modelado y Simulación, adscrito a la Escuela de Ingeniería de Sistemas e Informática de la UIS. Bucaramanga, Colombia. Mayor información sobre este trabajo y demás labores del grupo SIMON, http://simon.uis.edu.co/joomla/home/

2 Especialista en Educación con Nuevas Tecnologías de la Universidad Autónoma de Bucaramanga. UNAB. Es candidato a Magister en Ingeniería en Sistemas e Informática de la Universidad Industrial de Santander. Se ha desempeñado como docente de tecnología en el Colegio Aurelio Martínez Mutis de Bucaramanga, Colombia; Docente en la Especialización en Computación para la Docencia en la Universidad Antonio Nariño; Docente en la licenciatura en tecnología e informática en la Universidad Cooperativa de Colombia; ha sido docente cátedra en la escuela de física, en la escuela de sistemas e informática y en la escuela de metalúrgica en la Universidad Industrial de Santander. Actualmente colabora como co-investigador del Grupo SIMON de Investigaciones en Modelamiento y Simulación.

3 Magister en Informática de la Universidad Industrial de Santander, actualmente se desempeña como Profesor Titular de la Escuela de Ingeniería de Sistemas e Informática, en el área de Sistemas Dinámicos y Modelos de simulación, ha sido docente Invitado a varias Universidades a nivel nacional e internacional. Producto de sus investigaciones están MACMedia, micromundo para el aprendizaje de las ciencias en la educación media, basado en el modelo educativo centrado en los procesos de pensamiento; Evolución 3.5, herramienta software para la simulación con Dinámica de Sistemas. Sus publicaciones mas recientes son: Paisajes Dinámicos con HOMOS 1.0, en la Revista Iberoamericana de Informática Educativa; MACMedia”: Micromundo Para El Aprendizaje De Las Ciencias En La Educación Media. Un Enfoque Dinámico-Sistémico-“en el Quinto Congreso Iberoamericano De Informática Educativa; MICRHO: Herramienta Para El Desarrollo De Habilidades Del Pensamiento. Soportado En La Metodología De Modelamiento Basado En Objetos Y Reglas. En el Coloquio La Informática Y Los Jóvenes en Cuba; MACMedia 2.0”: Micromundo Para El Aprendizaje De Ciencias En La Educación Media" en el VI Congreso en Informática Educativa en Medellín; Proyecto MAC 1 a 11, Una Estrategia para Promover un Cambio en las Prácticas Educativas. En el Encuentro Iberoamericano de Formación Docente en Bogotá; Ponencia: "Propuesta de intervención en el proceso educativo para el mundo de Internet" en el VI Congreso Nacional de Informática Educativa. RIBIE Nodo Colombia. en Medellín; Ponencia: MAC 6-7 2.0: Micromundo para el Aprendizaje de la Ciencias de la naturaleza de sexto y séptimo grado. En el Primer Congreso Latinoamericano de Dinámica de Sistemas. Monterrey, México. 
Resumen: Este artículo presenta el análisis de experiencias, revisadas en diversos artículos, acerca de la implementación de la robótica en la educación, con el fin de obtener referentes pertinentes para la construcción de una propuesta para el aprendizaje de la robótica en la educación básica secundaria y media. Se consideran dos ámbitos de aplicación de la robótica en la educación: el primero es el aprendizaje de la robótica mediante la conceptualización de cada uno de los subsistemas de un robot para luego realizar la construcción de robots con diferentes propósitos, en este ámbito el objetivo es desarrollar las competencias, en los estudiantes, para el diseño y construcción de tecnofactos robóticos, mediante un proceso de construcción del conocimiento necesario para hacerlo; el segundo ámbito de aplicación es la utilización de la robótica como medio que motiva y da sentido a la construcción de conocimiento en diversas áreas, promoviendo habilidades y competencias en los estudiantes. Además, el aprendizaje de la robótica y el aprendizaje con robótica pueden ser mediados por la informática, la cual aporta tecnologías y herramientas software como los lenguajes de modelado matemático y simulación, tutoriales multimediales, laboratorios virtuales y remotos, entre otros. Así, este artículo se propone destacar cómo la robótica en el aula de clase permite, por una parte, el enriquecer estrategias de aprendizaje como apoyo a la formación integral de los estudiantes, y por otra, es un campo que presenta una demanda creciente en la atención de docentes e investigadores, así como en los estímulos para su investigación, desarrollo y divulgación de parte del estado.

Palabras claves: Educación, Informática Educativa, Robótica, Tic.

\begin{abstract}
This paper analyzes some experiences, previously reviewed in literature, about the use of robotics in education in order to obtain relevant models for the design of a proposal about robotics learning at Basic Secondary and Middle education levels. Two scopes of application of robotics in education are considered: firstly, learning robotics by defining the context of each robot subsystems and then creating robots for different purposes. The objective in this case is the development of skills for the design and construction of robotic artifacts, a process that implies the acquisition of the necessary knowledge to accomplish this task. The second scope of application is the use of robotics to encourage knowledge construction in different areas, fostering students' development ok skills and competences. Learning robotics and learning with robotics can be supported by computer science since this area provides software technology and tools such as Math Modeling and language simulation, Multimedia Tutorials, Virtual and Remote laboratories, among others. The purpose of this article is to highlight the importance of robotics in the classroom to enrich learning strategies which contribute to student's comprehensive education. This field of study has a growing demand by teachers and researchers, and stimulus to encourage its research, development and spread.
\end{abstract}

Keywords: Education, Computer uses in education, Robotics, Tic.

\title{
Introducción
}

La robótica es una de las expresiones de la tecnología cuya aplicación se ha extendido a diversos contextos de la vida del hombre. Además de sus diversas aplicaciones en la industria, hace presencia facilitando y mejorando actividades como: los vuelos no tripulados, el estudio del mundo submarino, la limpieza de piscinas, la exploración del espacio exterior con robots como el Opportunity, el Spirit, el Rocky IV, la Misión Robótica Juno y el Curiosity; en el ámbito del entretenimiento: la creación de robots, como Aibo de Sony que simulan características de una mascota, robots que pueden jugar fútbol, robots móviles, humanoides y muchos otros en los cuales se aplican los últimos adelantos tecnológicos en sonido, reconocimiento y síntesis de voz, e inteligencia artificial. 
En medicina, algunas de las aplicaciones se encuentran en robots que son teleoperados por médicos especializados, ubicados en cualquier parte del mundo y quienes realizan la intervención quirúrgica con precisión microscópica (microbiótica). Entre los beneficios obtenidos cabe mencionar: disminución de costos por desplazamiento del médico y la precisión en las acciones al eliminar ruidos como el temblor de las manos; en los desarrollos macrobióticos se resalta la construcción de prótesis que reemplazan eficientemente partes del cuerpo humano.

En el ámbito doméstico han sido creados robots programados para realizar diversas labores en la casa, liberando al ser humano de estas, en favor de aprovechar mejor su tiempo. Finalmente, los esfuerzos, en este contexto, se orientan en la creación de androides que puedan imitar al hombre en cuanto a su modo de andar o realizar acciones como la manipulación de objetos.

Por otra parte, como ha sido común en la historia del conocimiento, y en particular de la tecnología, los robots pueden presentar su lado negativo de acuerdo al uso de los mismos por el ser humano, como: los vuelos no tripulados para interferir en la privacidad de las personas, los exploradores espaciales para espiar países y los humanoides para llevar la guerra a nivel de robots armados y manipulados a distancia como se puede observar en los videojuegos (Bearden, 2009). Esto implica que al igual que ante las demás tecnologías, es necesario que la educación asuma de manera crítica, ética y responsable, su papel de formadora, difusora y enriquecedora de este campo del saber y el hacer humano.

La anterior apreciación de algunas de las aplicaciones de la robótica, deja ver la urgente necesidad de la educación de los jóvenes en el conocimiento, uso, análisis, adaptación, diseño y construcción de robots; la alfabetización para el buen uso de cada uno de estos tecnofactos; y la ineludible reflexión acerca de las ventajas y desventajas de su uso y adaptación a cada contexto social.

En este artículo se asume la revisión de literatura y experiencias de robótica en educación como un paso importante en la búsqueda de respuestas sobre cómo asumir la educación en robótica y con robótica, identificando qué tipo de instituciones educativas han implementado la robótica en el aula de clase, qué características tiene su uso y qué recursos se han utilizado, es decir, cómo se ha abordado el tema en los planes de estudio y cuáles han sido los objetivos y aprendizajes planteados.

Específicamente, el objetivo de esta revisión es contar con referentes que constituyan un punto de partida para el diseño de una propuesta pedagógica y metodológica para el aprendizaje de la robótica y con robótica en la educación básica secundaria y media, propuesta a generar y contextualizar en cada comunidad educativa, y a formular a partir de la experiencia en todos los niveles educativos, incluida la educación superior, donde las experiencias muestran más y mejores enseñanzas.

Esta búsqueda se orienta por dos perspectivas: el aprendizaje de la robótica y el aprendizaje con robótica; a su vez, estas dos miradas se conciben reconociendo la presencia de un contexto tecnológico, la informática, que facilita y potencializa las diferentes propuestas 
educativas en la escuela. El aprendizaje de la robótica se concibe en una dinámica de diseño, construcción y explicación de tecnofactos (Gallego-Badillo, 1998), es decir, en un ejercicio de creación y construcción de conocimiento. El aprendizaje con robótica se asume como el proceso de creación de un contexto (robótica - Informática) que dinamiza y da significado al aprendizaje colaborativo en las diferentes áreas del conocimiento escolar.

\section{El aprendizaje de la robótica y con robótica}

A mediados de los noventas, se inicia la utilización de diversos tipos de plataformas de aprendizaje apoyadas por robots, se diversifica la oferta de cursos en las universidades y colegios sobre robótica e igualmente, y en paralelo a esta actividad, se inicia un nuevo campo de investigación y desarrollo que ha tomado el nombre de Robótica educativa (Kumar, 2004). Al mismo tiempo, las empresas asumen el desarrollo de materiales de apoyo a las actividades en en el aula; ejemplo de esto son Lego (Lego MindStorms [en línea]), VexRobotics (VEX Robotics [en línea]) y los Ataos (Ata Epe [en línea]), quienes promueven una propuesta pedagógica para ciencia y tecnología del grupo de investigación "El aprendizaje y la enseñanza" de la Escuela Pedagógica Experimental en Bogotá, Colombia.

Sobre la robótica educativa, Jacek Malec (2001) realiza un análisis sobre su utilización en la educación clasificándola en dos tipos: "Robótica en educación y robótica para la educación". Los dos enfoques se presentan en este documento analizando el uso que se les da a los robots para el aprendizaje de la robótica y la utilización de la misma en el aprendizaje de temáticas en diversas áreas del conocimiento (Figura 1). Asimismo, se estudian las experiencias encontradas con el fin de determinar el papel que juega la informática en el aprendizaje de y con la robótica.

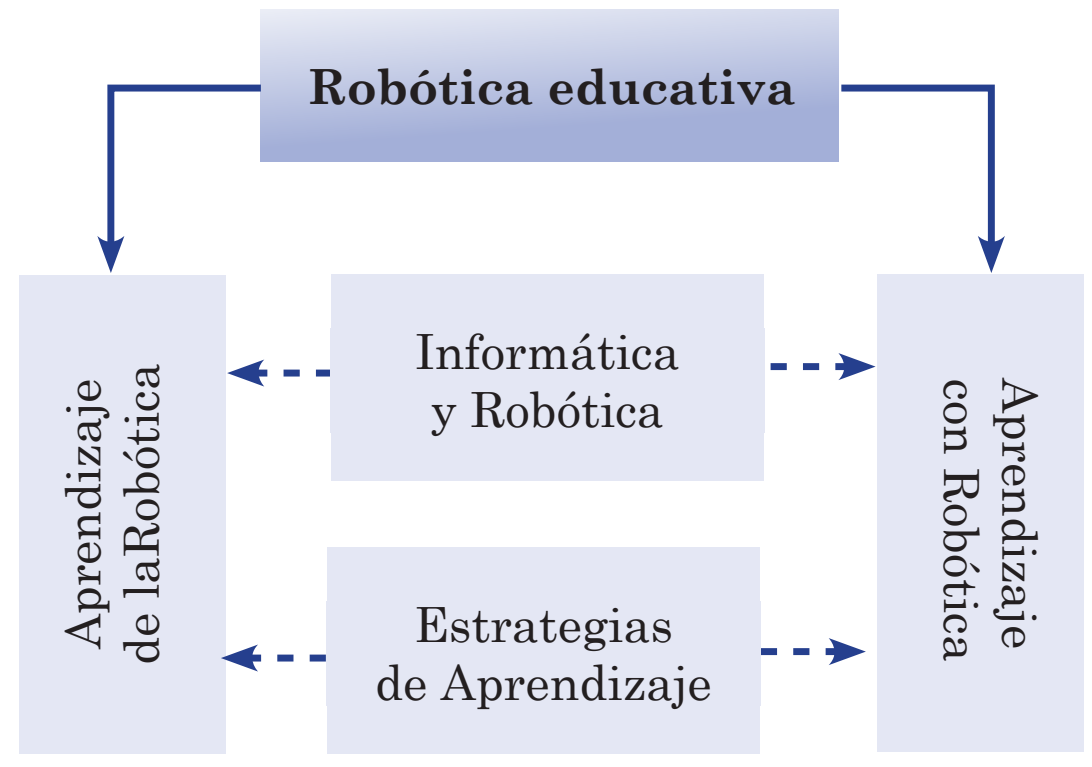

Figura 1. Robótica educativa. 


\section{El aprendizaje de la robótica}

La robótica, como tecnología que es, constituye el saber y el hacer sobre los robots, esto implica el uso del conocimiento de diversas áreas para el diseño, construcción, ensamble y puesta en funcionamiento de un robot con un fin específico. Asimismo, la robótica se constituye en la sinergia de los ejes de contenidos, contemplados en la educación en tecnología, como electricidad y electrónica, mecánica, energía, sensórica e informática.

Teniendo presente esta noción de robótica, el análisis de los diferentes artículos (Xudon y Weinberg, 2003) (Shuying, Zhao, et al., 2008) permite determinar los temas de robótica que el estudiante debe atender y que son aconsejables en una propuesta de aprendizaje de la robótica, como: historia, tipos de robots, sensórica, noción de realimentación y sistemas dinámicos, sistemas de control, automatización, ya que la robótica es una tecnología multidisciplinar (Maxwell y Meeden, 2000) (Smith, 1997), que aplica conocimientos de mecánica, inteligencia artificial, telecomunicaciones e informática, los cuales permiten el diseño y construcción de los robots.

El aprendizaje de las temáticas, de y para la robótica, puede lograrse desde dos puntos de partida diferentes: a partir de la conceptualización ${ }^{4}$ sobre robótica o a partir del diseño y construcción de aparatos robóticos, en los dos casos con el apoyo de software para el diseño y la simulación de los robots, y con la aplicación de estrategias de aprendizaje con el fin de lograr la motivación en los estudiantes para su estudio y aprendizaje (Figura 2).

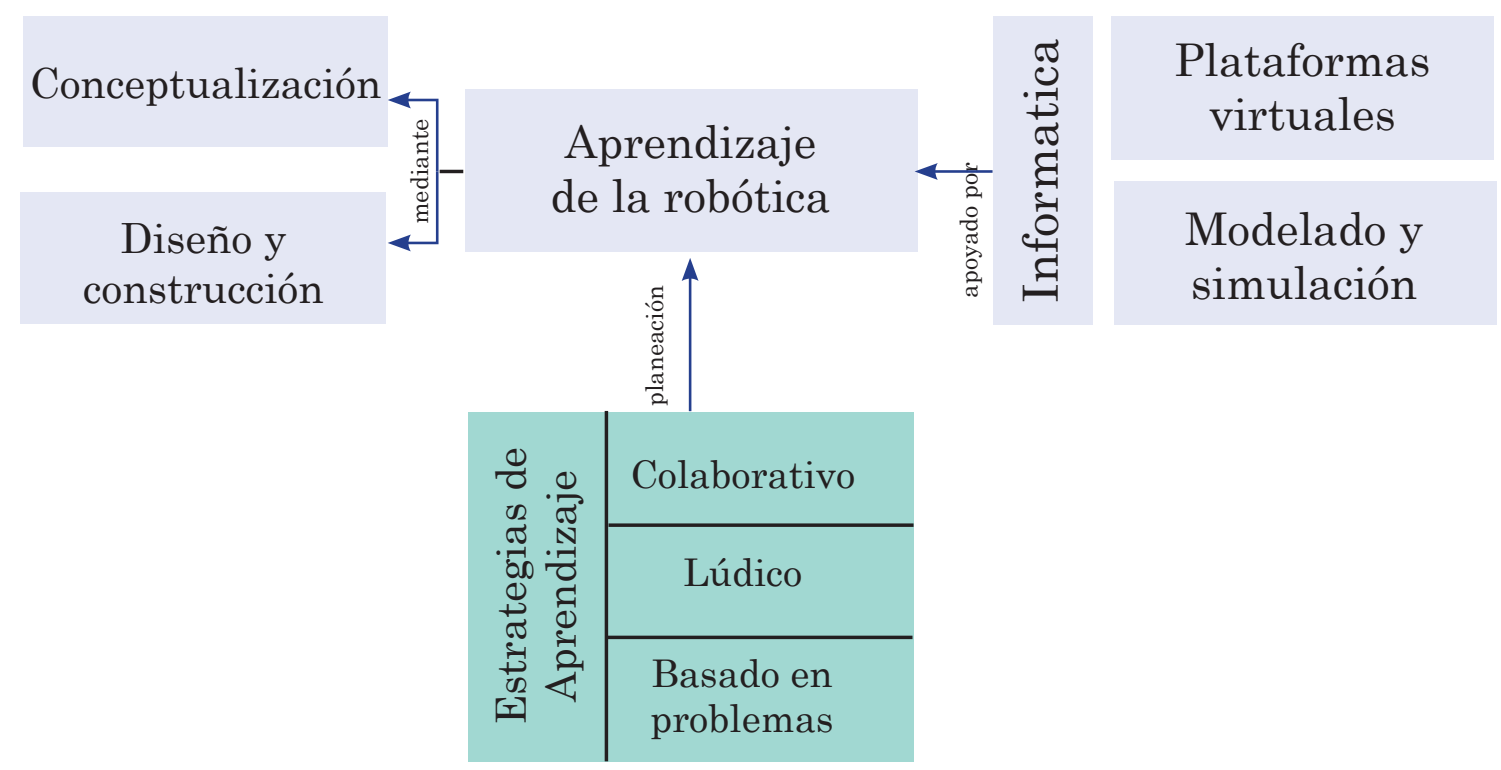

Figura 2. El Aprendizaje de la robótica

\footnotetext{
4 Se asume conceptualización como "el desarrollo o construcción de ideas abstractas a partir de la experiencia: nuestra comprensión consciente (no necesariamente verdadera) del mundo". Tomada de del libro "Mapas conceptuales. La gestión del conocimiento en la didáctica" de Virgilio Hernández Forte.
} 


\section{El aprendizaje sobre robótica a partir de la conceptualización}

El Instituto Norteamericano de Robótica (RIA, por sus siglas en inglés) define robot como "un manipulador multifuncional y reprogramable diseñado para mover materiales, piezas, herramientas o dispositivos especiales mediante movimientos programados y variables que permiten llevar a cabo diversas tareas" (Freedman, 1996).

En coherencia con Freedman, la propuesta de aprendizaje de la robótica debe contemplar el estudio de los diferentes elementos que conforman un robot y que se pueden considerar como los subsistemas del mismo (Lam, 2007). Así, son necesarios conocimientos acerca de los subsistemas de estructura y de movimiento del robot, los cuales constituyen la base para su funcionamiento y conllevan al estudio y aprendizaje sobre los diferentes componentes de la parte operativa y de la parte de mando del robot (Nourbakhsh, 2002). Igualmente, es necesario el estudio sobre los diferentes tipos de actuadores, sensores, transductores (Sklar, Parsons y Stone, 2004), y demás elementos que permiten la interacción del robot con el medio.

Los subsistemas de energía, sensórica, lógico, control y programación complementan el estudio de la robótica y permiten que, una vez construido, el robot atienda la necesidad o problemática para lo cual fue creado (Minguez, Javier, et al., 2005); y, durante el proceso de diseño, creación y puesta en funcionamiento, es posible el aprendizaje de temáticas de robótica, como manipulación del robot, visión por computador, inteligencia artificial y mecatrónica (Krotkov, 1996).

Para el aprendizaje de las temáticas de robótica, se han empleado kits creados para tal fin, como los elementos de Lego Mindstorms, una de las plataformas de Lego utilizadas en la educación. Galvan (2006) presenta la utilización de esta plataforma para el aprendizaje sobre diseño y control de sistemas de manipulación fijos, mediante el diseño, construcción y programación de un manipulador robótico. Igualmente, la universidad de Lund (Lund, Suecia) ofrece cursos a los estudiantes, utilizando el tablero base de LEGO 6.270, esta plataforma permite el control de motores, utilización de sensores y la programación para el control del robot con lo cual se facilita el aprendizaje de conocimientos básicos en robótica y métodos de diseño (Fiorini, 2005).

Con el mismo grado de importancia con el cual se selecciona la plataforma de trabajo, se debe determinar la estrategia a utilizar para involucrar a los estudiantes en el estudio de la robótica y la reflexión acerca de la ética en su desarrollo y utilización; con este fin existen estrategias muy creativas como comentar artículos sobre robótica tomados de revistas o periódicos recientes, ver videos y películas acerca de la robótica para luego realizar una reflexión sobre la realidad y la ficción de este tema (Piepmeier, Bishop y Knowles, 2003).

En una propuesta para el aprendizaje de la robótica, el determinar cómo se aprende concierne a la metodología de trabajo en clase: planear el conjunto de actividades, medios y recursos a utilizar, determinar el tiempo necesario para el desarrollo de cada actividad, determinar los objetivos de cada una de las áreas involucradas y el tema a trabajar, las estrategias más utilizadas para definir la metodología, en este caso, son el aprendizaje colaborativo (Barkley, Cross y Howell, 2007; Plestina, Turic y Papic, 2007; Ahlgren, 2002), 
aprendizaje lúdico (Jiménez, 2005; Maxwel, 2007; Minguez, Javier, et al., 2005; Murphy, 2001) y el aprendizaje basado en problemas (Escribano y del Valle, 2008; Narváez S. Carlos H. Leo, et al., 2006). Estas tres estrategias pueden ser combinadas de acuerdo a las competencias que se quieran promover, los contenidos que se vayan a desarrollar y los recursos que disponga la institución educativa.

En síntesis, la conceptualización en la robótica contempla la noción general de robot como resultado de la integración sistémica de cada uno de los subsistemas que lo constituyen, sin importar qué tipo de robot se esté estudiando; noción que a su vez facilita orientar y explicar una experiencia de diseño y construcción de un robot en particular. Además, la conceptualización está presente en el papel que juegan otras áreas de conocimiento para la explicación científica del funcionamiento del robot y para la selección adecuada tanto de plataformas robóticas que se puedan utilizar, como en la definición de la estrategia pedagógica que motive a los estudiantes a profundizar en el estudio de la robótica.

\section{El aprendizaje a partir del diseño y construcción}

Otro camino para el aprendizaje sobre robots es diseñando, construyendo y controlando un robot a medida que se estudian los conceptos necesarios para hacerlo; en este proceso se integran conocimientos, se materializa una idea y se aplica el conocimiento adquirido para justificar la utilización de cada elemento en el robot y explicar su funcionamiento (Jacek, 2001).

Son diversos los robots que se pueden construir: robots industriales, vehículos aéreos, terrestres y submarinos. En el periodo comprendido entre 1999 y 2004, en el laboratorio de sistemas robóticos de la Universidad de Santa Clara en Silicon Valley, Norte de California, equipos interdisciplinarios desarrollaron este tipo de proyectos de robótica y los grupos de estudiantes estaban conformados por jóvenes de diferentes niveles, programas y disciplinas académicas (Kitts y Quinn, 2004). Estas experiencias confirman que el aprendizaje de la robótica se desarrolla interdisciplinariamente y permite fomentar el trabajo en equipo y el aprendizaje colaborativo mediante la integración de equipos que desarrollan un trabajo de diseño, construcción y prueba de sus robots.

La integración del conocimiento se logra en un proceso construccionista como, por ejemplo, en la construcción de un robot pez (Xiaobo, 2006), que permite a los estudiantes un aprendizaje práctico, mientras realizan una investigación sobre comunicación inalámbrica, trabajando y aprendiendo sobre temáticas como fuerza, navegación, comunicación y sensórica, las cuales se integran en la construcción del robot cuyos fines son: trabajar en aguas hostiles, el monitoreo del medio ambiente y el control del mismo mediante un radio-control inalámbrico. Asimismo, en este ejemplo, se documenta la creación de una interfaz gráfica de usuario (GUI) para el monitoreo y control del robot (Turkle y Papert, 1992).

Al mismo tiempo que se construye la plataforma, se conceptualiza la robótica (Andueza y Aguirre, 2009); por ejemplo, Marco Polo, un juego de evasión y búsqueda en la que el robot perseguidor recibe información sobre la localización de los evasores en intervalos aleatorios de 
tiempo, es creado en una plataforma de trabajo donde los estudiantes logran el desarrollo de una herramienta que permite, mediante la lúdica, estudiar los conceptos de robótica (Perteet, McClintock y Fierro).

En el artículo titulado "Robots and Education in the classroom and in the museum" (Nourbakhsh, 2002), el autor describe la construcción de un robot insecto denominado Insect Telepresence, ubicado en la estación del Museo Carnegie de Historia Natural. Este tecnofacto les permite a los visitantes del museo ingresar al mundo de los insectos realizando un tour virtual, desde la perspectiva de un insecto pequeño, con explicaciones que facilitan el entendimiento de lo observado.

Maxwell (Maxwel, 2007) presenta su experiencia en la construcción de robots con la capacidad de interactuar con personas en entornos reales; este tipo de robots son desarrollados para participar en la exhibición y competencias robóticas de la Asociación Americana para la Inteligencia Artificial. La experiencia muestra la importancia de planificar el trabajo para alcanzar el objetivo final: la construcción de un robot robusto y el aprendizaje significativo que logran los estudiantes. Estas últimas experiencias nos permiten observar el referente del aprendizaje significativo que logra una propuesta de aprendizaje de los conceptos básicos de la robótica.

Igualmente, estas experiencias muestran el aprendizaje de la robótica, mientras se construye el robot, y en todas estas se encuentra como punto en común la necesidad de planear la construcción del robot siguiendo una determinada metodología. Por ejemplo, un equipo de la universidad de Texas (Lam, 2007) desarrolló el robot BlastyRas y los pasos del proceso implementado fueron: la evaluación de las necesidades, el diseño básico del robot, la construcción de un primer prototipo y la evaluación del diseño; este último paso puede llevar a la reconstrucción o a la revaloración de las necesidades y repetir el proceso hasta obtener el producto final (Figura 3).

Design Secuence

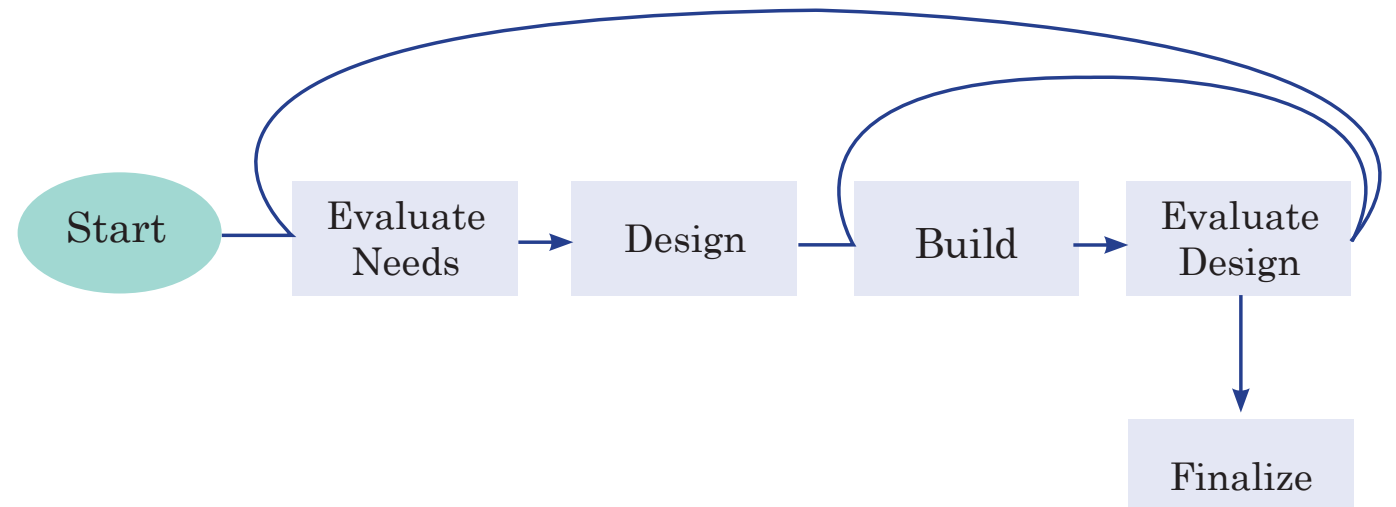

Figura 3. Diagrama de flujo del proceso de construcción del robot, tomado de (Lam, 2007). 
El proceso de diseño y construcción involucra la gestión de los recursos necesarios para la construcción de los robots, algunos son elementos básicos como motores, sensores, dispositivos electrónicos, los cuales pueden obtenerse mediante el reciclaje de partes de computadores, impresoras, scanners, etc..., (Mirats y Pfeifier, 2006). Mientras que la parte de control requiere circuitos más complejos donde se aplican conocimientos de electrónica para el diseño del circuito, la elaboración del impreso, el montaje de los elementos y la verificación de su funcionamiento.

La propuesta de aprendizaje de la robótica debe definir elementos básicos, necesarios para desarrollar en los estudiantes competencias tales como: la toma de decisiones basadas en el conocimiento, el formular explicaciones científicas y el trabajo en equipo. Estos elementos básicos deben determinarse dando espacio para que, de acuerdo a la capacidad de adquisición de recursos por parte de la institución, se lleve a cabo construcciones, desde las más simples pero básicas en el proceso, como las más sofisticadas que los medios lo permitan, mediante el trabajo con los elementos básicos logrados con el reciclaje de partes electro-mecánicas o mediante la adquisición de kits de robótica existentes en el mercado.

A manera de conclusión, el aprendizaje de los conceptos básicos de robótica puede darse durante el proceso para construir un robot; a nivel universitario este objetivo se logra integrando equipos interdisciplinarios de ingeniería de sistemas, electrónica y mecánica de diferentes niveles y programas. En los niveles de básica secundaria y media se debe propender por el desarrollo de competencias de trabajo en equipo, toma de decisiones basadas en el conocimiento y la explicación científica de los tecnofactos construidos.

Además, el robot es construido siguiendo un proceso determinado de planeación, construcción y verificación y es posible, mediante estos proyectos, promover y aplicar el pensamiento sistémico, al contemplar cada uno de los elementos que conforman el robot, su función individual y la sinergia alcanzada en el funcionar del todo construido. Igualmente, es posible hacer explícita la relación: experiencia de construcción-explicación científica del funcionamiento del objeto construido, superando la barrera de la mera formación de ensambladores de tecnofactos, para llegar al nivel de la aplicación de los conocimientos de tecnología, apoyados por teorías científicas, para el diseño y construcción de soluciones creativas a problemas significativos en la cotidianidad del estudiante.

\section{El aprendizaje con robótica}

La robótica en el aula permite, además de estudiar tópicos de automatización y control de procesos del área de tecnología e informática, el aprendizaje de temas de diferentes áreas de conocimiento, dado el interés que despierta el trabajar con objetos concretos y llamativos como un robot y, si se implementa, junto con los recursos, una metodología y una adecuada planificación, se estimula en los estudiantes el aprendizaje de temáticas que, de otra forma, sería más difícil de entender y poco motivantes para su estudio.

Lo anterior justifica el uso de robots en el aula para el aprendizaje de conocimientos de diferentes áreas (Pinto, Barrera y Perez, 2010), de esta forma, una propuesta de robótica 
educativa para la educación básica secundaria y media debe implementarse bajo un enfoque pedagógico que tenga en cuenta el ambiente de aprendizaje, la planeación de las actividades, los recursos, el tiempo necesario para la realización de cada una de estas y la metodología con la cual se va a desarrollar la labor (Figura 4). Estas características del modelo pedagógico garantizarán la construcción y reconstrucción de conocimiento por parte del estudiante.

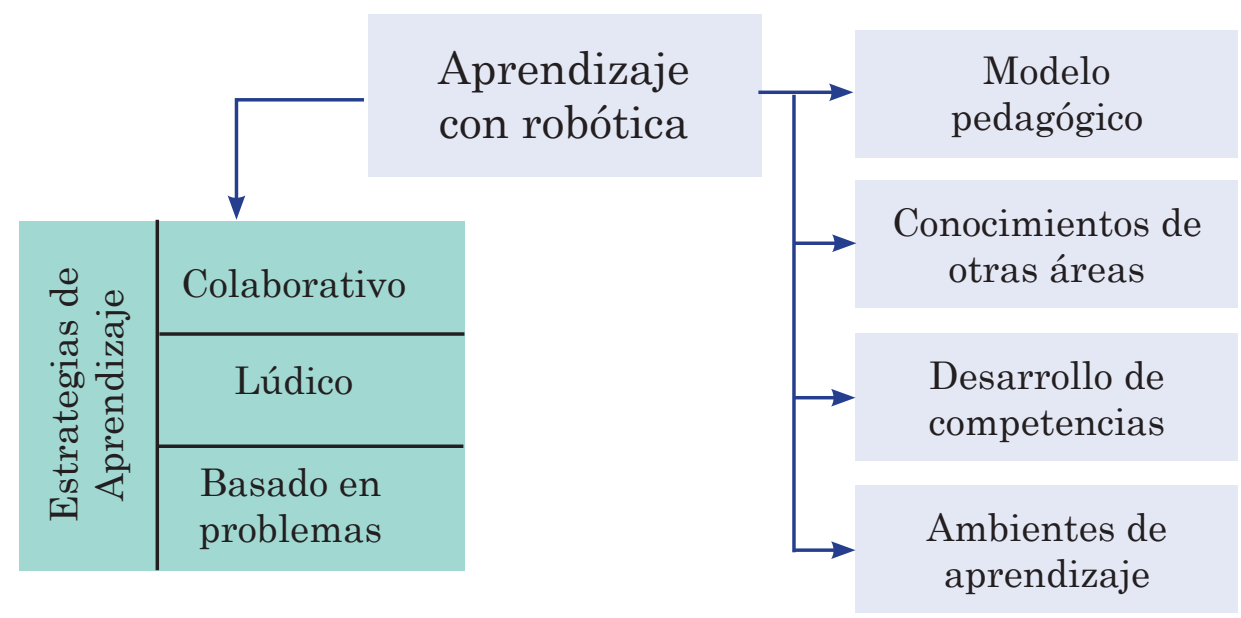

Figura 4. El aprendizaje con la robótica

\section{El enfoque pedagógico}

En general, el aprendizaje con robótica se basa en la estrategia del aprendizaje por proyectos y se logran avances en la capacidad de diseño y planeación, en el trabajo en equipo y en la resolución de problemas; asimismo, aporta en el desarrollo de la creatividad de los participantes del proyecto.

Las actividades generadas para la construcción de conocimiento utilizando la robótica se caracterizan por la aplicación de teorías pedagógicas como el constructivismo de Piaget (Pitti, curto y Moreno, 2010; Turkle y Papert, 1992; Miglino, Lund y Cardaci, 1999; Bers y Urrea, 2000), esta teoría propone que el conocimiento no es simplemente transmitido por el profesor al estudiante, sino activamente adquirido por quien aprende, los niños no reciben ideas, ellos las elaboran; y el construccionismo de Papert, (Xudong y Weinbeg, 2003), que sugiere que quienes aprenden están particularmente motivados cuando viven la experiencia de construir, ya sea un robot, un tecnofacto, un poema, un castillo de arena, un programa de computador o una teoría científica, sobre lo cual puedan reflexionar y compartir con otros. En síntesis, como lo dice Papert (1995): "el construccionismo, mi personal reconstrucción del constructivismo, tiene como principal característica que observa la idea de la construcción mental más cerca que los otros -ismos educativos. Le concede una especial importancia al papel que pueden desempeñar las construcciones en el mundo como apoyo a las que se producen en la cabeza, convirtiéndose así en una doctrina menos mentalista”. La aplicación de estas teorías de 
aprendizaje tiene como propósito desarrollar el pensamiento sistémico (Andrade, 2007), el desarrollo cognitivo, el desarrollo del pensamiento científico y la capacidad creativa e investigativa en los estudiantes.

Asumiendo la orientación pedagógica de Papert, la docente Ana Acuña (2006) expone que la formulación de proyectos de enseñanza y aprendizaje involucrando la robótica como motor de innovación, facilita el lograr un cambio en la forma de pensar y actuar tanto de los profesores como de los estudiantes. En su investigación, esta docente resalta la importancia de los recursos tecnológicos en el desarrollo de los proyectos, haciendo un especial énfasis en las metas a alcanzar, es decir, en las capacidades cognitivas, sociales y tecnológicas que se esperan alcanzar al realizar el proyecto y el grado de comprensión que deben lograr los estudiantes.

En esta experiencia (Acuña, 2006), se aplica la teoría de aprendizaje del construccionismo y utiliza la estrategia del aprendizaje por proyectos donde los estudiantes, de los 3 ciclos de la Enseñanza General Básica (EGB) de Costa rica, diseñan, construyen y programan prototipos de solución a los problemas de investigación propuestos, alcanzando logros en el diseño y la creación de prototipos, la capacidad de trabajo en equipo y el diseño de sistemas de control automatizado.

Para promover el enfoque pedagógico, la informática aporta el uso de herramientas tecnológicas, por ejemplo, la manipulación de robots móviles en ambientes virtuales, como en Pérez (2004), donde se realiza la experiencia con estudiantes desde los 5 hasta 12 años de edad, con el fin de promover en ellos los diferentes tipos de pensamiento y su desarrollo cognitivo aplicando teorías de aprendizaje como el constructivismo, la holística y la metacognición. Asimismo, se destaca la importancia de la robótica en la integración de las diversas áreas del conocimiento y la aplicación del pensamiento sistémico para la creación de entornos de aprendizaje que proporcionen un alto grado de motivación; así, el trabajo con robótica permite de forma dinámica y motivante la construcción de conocimiento en áreas como matemática, ciencias, sociales y tecnología, entre otras.

En cuanto a los recursos físicos de robótica para trabajar en el aula, están siendo desarrollados por empresas como Lego, donde se han creado: el tablero base de Lego 6.270, Lego robots, Lego MindStorms, Lego NTX y Topobo. El trabajo con estos recursos permite el aprendizaje de temas de diversas áreas a nivel universitario como inteligencia artificial, agentes inteligentes, visión por computador (Plestina, Turic y Papic, 2007) y lenguajes de simulación. De esta manera, la robótica permite la adquisición de conceptos básicos de cada una de las áreas y, a la vez, el estudiante observa su aplicación, por ejemplo, para el estudio del hardware y el software de un sistema de control (Smith, 1997).

Este tipo de aplicaciones debe tomarse como referencia para determinar los aprendizajes que pueden lograrse, guardando las proporciones de profundidad y rigurosidad, en el nivel de educación básica secundaria y media. Por ejemplo, en áreas como biología, Miglino y su grupo de trabajo de Lego (Miglino, Lund y Cardaci, 1999) desarrollaron una aplicación de robots que simulan organismos artificiales que se transforman en elementos de enseñanza aplicando las teorías de Piaget. Los estudiantes construyeron robots que simulaban el comportamiento 
animal, lo que permitía verificar y construir conocimientos de la teoría de la evolución de Darwin, manipulando los procesos de selección, reproducción y mutación y las características de mente-cuerpo del robot construido.

A nivel de educación básica y media, teniendo en cuenta experiencias de Centro y Suramérica, la robótica se utiliza para verificar y promover formación en ética moral. Bers y Urrea (2000) presentan el programa Con-ciencia, el cual se desarrolla utilizando kits de robótica de Lego Mindstorms y planteando la integración de la religión y el aprendizaje de la tecnología, se alcanzan los objetivos mediante el trabajo conjunto de estudiantes, padres de familia y profesores. Otro ejemplo de integración de conocimiento de las ciencias, la informática y la tecnología se visualiza en un proyecto de estudio del medio ambiente y la energía solar (Narváez S. Carlos H. Leo., et al., 2006), mediante la construcción de un robot para medir el grado de contaminación ambiental en un sector de la ciudad de Bogotá, Colombia, logrando así, que la robótica proporcione una real integración de las áreas de conocimiento.

Aprendizajes en matemática como los números, la geometría, los colores y la motivación por el aprendizaje de la robótica, se ha logrado mediante la utilización de un robot de Lego Mindstorm, en diversos talleres realizados con niños de nivel de preescolar y primaria, en el departamento de Boyacá, Colombia (Pinto, Barrera y Pérez, 2010). En Yucatán, Mexico, se enseña el plano cartesiano mediante un robot Lego y scratch, realizando los ejercicios de posicionamiento en el plano mediante la programación del robot y la animación de un objeto en la pantalla del computador (Tec, Uc, Gonzalez, Garcia, Escalante y Montañez, 2010).

Es posible entonces utilizar la robótica como elemento motivador e integrador del conocimiento de las diferentes áreas y para esto son útiles diversas plataformas robóticas que se consiguen en el comercio o mediante la construcción del robot con elementos nuevos o de reciclaje. Se aprecia que la motivación lograda en los niños y jóvenes es posible extenderla a los padres de familia, al desarrollar actividades que los acerquen a la tecnología y aprender algunos temas, apoyados en sus hijos (Bers y Urrea, 2000).

La robótica como medio o contexto de una educación significativa, facilita a los niños y jóvenes, superar barreras de aprendizaje al despertar su interés y, asimismo, los apoya en el proceso de construir y reconstruir el conocimiento. Además, aporta a la formación de los niños con necesidades individuales especiales, lo que permite la temprana identificación de la problemática y la forma de ayudar a superarla (Virnes, 2008); asimismo, se puede lograr la inclusión de sectores marginados, en el aprendizaje y uso de este tipo de tecnologías (Castro y Acuña, 2012).

En la formación, el desarrollo de actividades con robótica en el aula facilita promover en los estudiantes las competencias para indagar, seleccionar, asumir críticamente y adaptar tecnologías, de tal forma que se dé la discusión sobre la utilización ética de estos desarrollos tecnológicos, el beneficio y bienestar que proporcionan como elementos creados para satisfacer necesidades humanas, e igualmente lo contraproducente, el deterioro y el daño que pueden causar al ser creados o utilizados para propósitos no éticos. 


\section{Ambientes de aprendizaje con la robótica}

La utilización de plataformas robóticas, equipos de hardware y software instalados con un fin específico y que posibilitan la creación de entornos de aprendizaje cuyo objetivo principal es la construcción y programación del robot, facilitan la realización de las actividades determinando desde el inicio el tipo y la cantidad de recursos necesarios para lograr los aprendizajes esperados en cada una de las experiencias en el aula de clase.

El aprendizaje de diferentes áreas de conocimiento se ve fortalecido con el desarrollo de plataformas robóticas a bajo costo que permiten su adquisición por las instituciones educativas (Muñoz, Andrade y Londoño, 2006) y, con estas, la diversificación del tipo de experiencias que son ejecutadas en el aula. Por ejemplo, Weinberg y Xudong presentan experiencias de trabajo con Lego RCX, plataforma desarrollada en el MIT, una aplicación de la misma plataforma Lego la presenta Zhao Shuying, trabajando el movimiento y posicionamiento de un robot (Shuying, Zhao, et al., 2008).

Otra aplicación, del desarrollo de hardware y software para plataformas robóticas, permite el uso de sistemas dinámicos para el aprendizaje de la física facilitando el estudio de conceptos de masa, inercia y fricción, entre otros, gracias al modelado y análisis de sistemas dinámicos (Cox, 2008). Estos sistemas incluyen plataformas modulares dotadas de diferentes tipos de sensores que son utilizados para la adquisición de datos que, luego, son procesados en aplicaciones de Matlab.

Igualmente, estas plataformas robóticas son utilizadas con propósitos formativos y en el desarrollo de los cursos se proponen objetivos tanto educativos como de investigación (Maxwell y Meeden, 2000). Algunas de estas han sido creadas para los diferentes niveles escolares y otras para trabajar áreas específicas de conocimiento, pero el éxito de su utilización depende de la formación que se dé a los docentes para la planeación y ejecución de actividades que las incluyan en la construcción de conocimiento en las diversas temáticas del área en estudio.

El cumplimiento de esta tarea requiere de transformaciones profundas en el sistema escolar, iniciando en los Ministerios de Educación, que deben dar solución a tres problemas fundamentales: i) debido a la falta de lineamientos pedagógicos y metodológicos que orienten a los docentes sobre el aprendizaje de y con robótica, no existen estándares para el área de tecnología e informática, ni se encuentran propuestas estructuradas para trabajar la robótica en la escuela, ii) la necesidad de formación docente para utilizar los recursos y aplicar una determinada metodología para el aprendizaje de los conceptos básicos de robótica, y iii) la falta de infraestructura y recursos; se necesita dotar las instituciones educativas de los recursos necesarios para poder implementar sistemas y llegar hasta la automatización de los mismos, ya que las instituciones educativas se encuentran con el gran inconveniente de los altos costos de los elementos necesarios para implementar la automatización de procesos.

\section{Informática y robótica}

La robótica no ha sido ajena a la utilización de la tecnología de la información en su desarrollo y aplicación (Uldemolíns, 2008); en el aprendizaje de y con robótica se encuentra 
desde simuladores de los movimientos de los robots, tutoriales multimediales, laboratorios virtuales y remotos, hasta la utilización de internet y el compartir recursos a través de las redes de computadores (Figura 5).

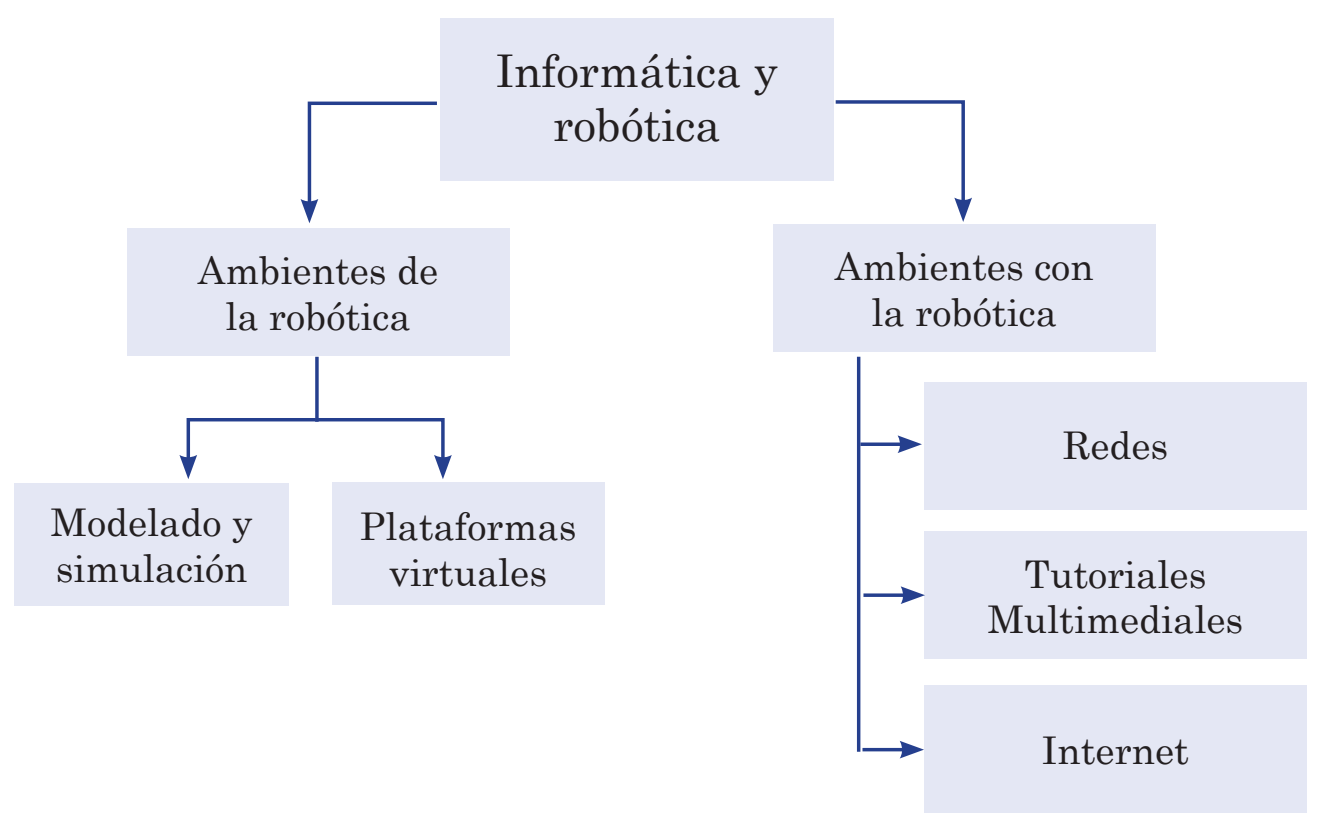

Figura 5. Informática y robótica

El computador y la ingeniería de software hacen presencia, de diversas maneras, en la educación y la línea de desarrollo de software para la educación ha permitido la creación de aplicaciones de simulación y tutoriales que facilitan el estudio del componente mecánico del robot; la producción, transmisión y transformación de su movimiento es recreada mediante software (Raz, 1989). Los estudiantes requieren de menos tiempo para el aprendizaje sobre movimientos del robot utilizando simuladores como GRS (Graphic Robot Simulator) y la creación de interfaces gráficas de usuario (GUI) para el monitoreo y control del robot (Xiaobo, 2006), laboratorios virtuales y el uso de software multimedial (Cosma, Confente, Botturi y Fiorini, 2003), para el aprendizaje de la dinámica y el control de procesos (Ortiz, Rennola y Johnny, 2005).

Algunas de las técnicas informáticas aplicadas en el aprendizaje de la robótica, como la simulación de procesos mediante software, es utilizada, en la Universidad de Buenos Aires, Argentina, en el Laboratorio de Informática Educativa y Medios Audiovisuales (Odorico, Lage y Cataldi, 2009), donde se desarrolla un proyecto de software para la educación que permite, utilizando conceptos de simulación, que los estudiantes puedan diseñar un brazo de robot ingresando las características elementales del mismo, como tamaño del brazo y del antebrazo, el tipo de articulaciones y los grados de giro a la derecha y a la izquierda. 
Otra aplicación de la informática, el desarrollo de plataformas virtuales para la educación en robótica, es una de las soluciones a los problemas de los sistemas robóticos físicos, como costos, disponibilidad de equipos, complejidad técnica, flexibilidad del entorno de trabajo y limitaciones de tiempo. Estas plataformas permiten la investigación en campos como realidad virtual y diseño asistido por computador aplicados al modelado y la simulación virtual (Demetriou y Lambeert, 2005), además, este tipo de plataforma permite a los estudiantes hacer prácticas de robótica móvil diseñando algoritmos y probándolos en robots reales (Payá, Reinoso, Gil y Jiménez, 2007), utilizando estos recursos en el desarrollo de ambientes para la educación a distancia (Carusi, F. [et. al], 2004). Es necesario analizar y determinar cuáles de estas aplicaciones pueden utilizarse en la educación básica secundaria y media, para fortalecer el aprendizaje de la robótica o con robótica, en educación presencial o a distancia.

El diseño, desarrollo y utilización de software para el aprendizaje de conceptos en diversas áreas de conocimiento (Vásquez, 2006) tiene una aplicación, en el área de la física, mediante el modelado de plataformas robóticas con capacidad de adquirir, analizar y controlar información, permitiendo el aprendizaje y la aplicación de conocimientos de física en temáticas de óptica, ondas de sonido, leyes de los gases y ultrasonido (Kocijancic, 2000). Junto con el software, se desarrolla también elementos de hardware que posibilitan su utilización. Danahy y otros (Danahy, Goswamy y Rogers, 2008) logran el diseño de computadores portátiles que involucran un software interactivo cuyo objetivo es crear un puente entre currículo, ambientes de programación y plataformas de robótica, que le faciliten al docente la tarea del aprendizaje de la robótica y lo liberen de la presión de ser expertos en áreas como programación, robótica y estrategias de aprendizaje.

Asimismo, las redes de computadores, desde sus inicios, fueron creadas para compartir recursos y el desarrollo de laboratorios de acceso remoto (Galvan, S., et al., 2006) y, gracias a estas ventajas, han sido la solución a la problemática de los altos costos de materiales (Coelho, Paulo, et al., 2007), ya que proveen la infraestructura para montar y modificar experiencias, de tal forma que es posible compartir recursos de dos instituciones logrando reducir costos y aumentando la cantidad y tipos de experiencias para los estudiantes como se aprecia en la experiencia de Netrolab, un laboratorio remoto creado conjuntamente por "The University of Reading" y "the University of Nottingham" (Mckee y Barson, 1997).

\section{Conclusiones}

El desarrollo de metodologías y propuestas pedagógicas para el aprendizaje de la robótica contempla tanto lo tecnológico que permita la utilización de tecnofactos facilitadores del trabajo del docente, como lo pedagógico en la aplicación de enfoques y estrategias pedagógicas que permitan logros coherentes con los requerimientos y exigencias del sistema educativo.

El uso de la tecnología de la información permite la mediación de una herramienta tan versátil como lo es el computador, al utilizarlo como el intermediario capaz de viabilizar estrategias de aprendizaje, mediante la utilización de un software que logre motivar a los estudiantes para el uso de los recursos y resolver, con ellos, retos mediante la utilización de la tecnología de la robótica. 
Una propuesta de educación en robótica puede facilitar el desarrollo de competencias ciudadanas y laborales específicas, que garantice superar el nivel de los simples ensambladores que se limitan a seguir instrucciones para obtener el tecnofacto final, para promover en el estudiante el desarrollo de las competencias para la toma de decisiones basadas en el conocimiento y la explicación científica del funcionamiento del tecnofacto construido, logrando así un aprendizaje de la robótica y una formación científica y tecnológica del educando.

Los conocimientos y técnicas de la informática brindan la oportunidad de disponer de plataformas dinámicas de aprendizaje que facilitan la construcción de conocimiento mediante actividades presenciales o virtuales. El uso de estas plataformas posibilitan el compartir recursos entre instituciones, mitigando uno de los grandes problemas de los proyectos de educación en robótica: el costo de los materiales.

Para consolidar una comunidad docente en robótica en la educación, se hace necesario sistematizar y divulgar las experiencias en esta área y así forjar el camino que facilite a nuevos docentes incluir actividades en el aula de clase, reformar los planes de estudio y modificar el currículo, de tal manera que se aproveche este recurso que constituye un elemento de motivación para el aprendizaje de los estudiantes.

El aprendizaje de y con robótica, en el contexto de la tecnología de la información, constituye dos alternativas que pueden integrarse para la construcción de un proyecto educativo de aprendizaje significativo y de formación de científicos.

La propuesta pedagógica y metodológica de aprendizaje de y con robótica debe contemplar los elementos necesarios de un modelo educativo determinando sus fines, los contenidos a contemplar, la metodología y estrategias de aprendizaje, los recursos físicos mínimos para el desarrollo de las actividades y los criterios de evaluación que permitan valorar el grado de desempeño de los estudiantes. Además, la propuesta debe visualizar la forma en como la informática puede apoyar las actividades de aprendizaje de y con robótica.

\section{Agradecimientos}

Los autores: Pedro Antonio López Ramírez, magister en Ingeniería de Sistemas e Informática, y Hugo Andrade Sosa, profesor titular de la Universidad Industrial de Santander (UIS) - Colombia y Director del Grupo SIMON de Investigación, agradecen a la Universidad Industrial de Santander (UIS) y a su Escuela de Ingeniería de Sistemas e Informática por mantener el programa de Maestría en Informática y en este, el espacio de investigación que permitió la labor investigativa que sustenta lo planteado en el presente artículo.

\section{Referencias bibliográficas}

Acuña, A. (2007). La Robotica Educativa: Un Motor para la Innovación. Recuperado de http://www.fod.ac.cr/robotica/descargas/roboteca/articulos/2007/roboticamotor innova articulo.pdf 
Ahlgren, D. (2002). Meeting educational objectives and outcomes through robotics education. World Automation Congress, 2002. Proceedings of the 5th Biannual, 14, 395-404.

Alvarado, G. (2007). El concepto de competencia en la perspectiva de la educación superior. foro El concepto de competencia: su uso en educación. Bucaramanga. Recuperado de http://groups.google.com/group/alianza-agro/web

Andrade, H. H., y Gómez, L. C. (2009). Tecnología Informática en la Escuela. Bucaramanga: UIS.

Andrade, H. (2007). Pensamiento Sistémico: Diversidad en búsqueda de Unidad. Bucaramanga: Ediciones Universidad Industrial de Santander.

Andueza, L., y Aguirre, I. (2009). Diseño de un manipulador robótico con tres grados de libertad para fines educativos. Revista Ciencia e Ingeniería., 3-14.

Ata Epe [en línea]. (s.f.). Recuperado de http://roboticaescolar.net/Ataos

Barkley, E., Cross, P., y Howell, C. (2007). Técnicas de Aprendizaje Colaborativo. Madrid: Ediciones Morata.

Bearden, T. (2009). PBS Newshour. Recuperado de http://www.pbs.org/newshour/bb/science/ jan-june09/robots 04-23.html

Bers, M. y Urrea. C. (2000). Technological prayers: Parents and children working with robotics and values. Robots for kids, 93-217.

Carusi, F., et al. (2004). Distance learning in robotics and automation by remote control of Lego mobile robots. Robotics and Automation, Proceedings. ICRA '04. IEEE International Conference on, 2(1), 1820-1825.

Castro, M., y Acuña, A. (2012). Propuesta comunitaria con robótica educativa: valoración y resultados de aprendizaje. TESIS, 91-119.

Coelho, Paulo, et al. (2007). A Web Lab for Mobile Robotics Education. IEEE International Conference on Robotics and Automation.

Complubot [en línea]. (s.f.). Recuperado de Memoria de actividades del curso 2007/08: http:// complubot.educa.madrid.org/inicio.php?seccion=principal

Cosma, C., Confente, M., Botturi, D., y Fiorini, P. (2003). Laboratory tools for robotics and automation education. 3303 - 3308.

Cox, D. (2008). Hands-on experiments in dynamic systems and control for applied education in robotics and automation. Automation Congress, 2008. WAC 2008. World. 1-6. 
Danahy, E., Goswamy, A., y Rogers, C. (2008). Future of robotics education: The design and creation of interactive notebooks for teaching robotics concepts. Technologies for Practical Robot Applications, 2008. TePRA 2008. IEEE International Conference on, 131 - 136.

Demetriou, G., y Lambeert, A. (2005). Virtual environments for robotics education: an extensible objectoriented platform. Robotics \& Automation Magazine, IEEE, 12(4),75 - 91.

Escribano, A., y Del Valle, Á. (2008). El Aprendizaje Basado en problemas. Madrid, España: Narcea Ediciones.

Fiorini, P. (2005). LEGO kits in the lab [robotics education]. Robotics \& Automation Magazine, IEEE, $12(4), 5$.

Freedman, A. (1996). Diccionario de computación (7 ed.). México: Mcgraw - Hill.

Gallego-Badillo, R. (1998). Discurso Constructivista sobre las tecnologias, una mirada epistemologica. Bogotá: MAGISTERIO.

Galvan, S. (2006). Innovative robotics teaching using LEGO sets. Robotics and Automation, Proceedings 2006 IEEE International Conference,721 - 726.

Galvan, S., et al. (2006). Innovative robotics teaching using LEGO sets. Robotics and Automation, 2006. ICRA 2006. Proceedings 2006 IEEE International Conference :721 - 726.

Gualdrón, L., Barbosa, J., y Vasquez, C. (2010). La perspectiva semiótica como base para la construcción curricular. Una apuesta de la UIS hacia la Formación Regional en Agroindustria. Revista de Pedagogia, 31(89), 277 - 306.

Jacek, M. (2001). Some thoughts on robotics for education. Proceeding of American Association of Artificial Intelligence Symposium on Robotics and Education. Lund University.

Jiménez, C. (2005). La inteligencia Lúdica (1a ed.). Bogota D.C., Colombia: Editorial Magisterio.

Kitts, C., y Quinn, N. (2004). An Interdisciplinary Field Robotics Program for undergraduate Computer Science and Engineering Education. ACM Journal on Educational Resources in Computing, 4(2), 1- 22.

Kocijancic, S. (2000). Computerized laboratory practice for future science and technology teachers. Frontiers in Education Conference, 2000. FIE 2000. 30th Annual,1, 13- 18.

Krotkov, E. (1996). Robotics laboratory exercises. Education, IEEE Transactions, 39(1), 94- 97.

Kumar, D. (2004). Introduction to Special Issue on Robotics in Undergraduate Education. ACM Journal on Educational Resources in Computing, 4(2). 
Lam, C. (2007). BlastyRAS. The IEEE Robotics and Automation Society (RAS) at The University of Texas at Austin Proudly.

Lego MindStorms [en línea]. (s.f.). Recuperado de http://mindstorms.lego.com/en-us/Default. aspx

Maxwel, B. (2007). "Building robot systems to interact with people in real environments. Autonomous Robots, 22(4), 353-367. doi. 10.1007/s10514-006-9020-9

Maxwell, B. A., y Meeden, L. A. (2000). Integrating Robotics Research with Undergraduate Education. Intelligent Systems and Their Applications, IEEE, 15(6), 22-27.

Mckee, G., y Barson, R. (1997). NETROLAB: a networked laboratory for robotics education. Robotics and Education, IEE. Colloquium on, 8, 1-3.

MEN. (2006). Estándares básicos de competencias en lenguaje, matemática, ciencias y ciudadanas: Lo que los estudiantes deben saber y saber hacer con lo que aprenden. (M. D. NACIONAL., Ed.), Colombia: Revolución Educativa.

Miglino, O., Lund, H., y Cardaci, M. (1999). La robótica como herramienta para la educación. Journal of Interactive Learning Research, 10(1), 25-47.

Minguez, Javier, et al. (2007). Silla de ruedas inteligente controlada por voz. Primer Congreso Internacional de Domótica, Robótica y Teleasistencia para Todos.

Mirats, J., y Pfeifier, C. (2006 ). Mobile robot design in education. Robotics \& Automation Magazine, IEEE, 13(1), 69-75.

Muñoz, N. D., Andrade, C. A., y Londoño, N. (2006). Diseño y construcción de un robot móvil orientado a la enseñanza e investigación. Ingeniería y Desarrollo, 19.

Murphy, R. (2001). Competing for a robotics education. Robotics \& Automation Magazine. IEEE. 8(2), 44-55.

Narváez S. Carlos H. Leo., et al. (2006). Ntics Robótica y Energía Solar. VIII Congreso Colombiano de Informática Educativa.

Navas Garnica, X. M. (2006). Propuesta Informática para la Educación en el Cambio, Basada en Ambientes de Modelado y Simulación. Un enfoque Sistémico. Bucaramanga.

Nomura, T., et al. (2007). Implications on Humanoid Robots in Pedagogical Applications from Cross-Cultural Analysis between Japan, Korea, and the USA. Robot and Human interactive Communication. 13th IEEE. International Workshop, 1052-1057. 
Nomura, T., et al. (2008). Robotics in education: Psychological relationships with "makingartifacts," computers, and mathematics in Japan. (I. I. Conference, Ed.) Intelligent Robots and Systems, 3668- 3673.

Nomura, T. y Kanda, S. (2004. ). Experimental Investigation into Influence of Negative Attitudes toward Robots on Human Robot Interaction. Proc. 3rd Workshop on Social Intelligence Design (SID 2004)., 125-135.

Nourbakhsh, I. (2000). Robots and Education in the Classroom and in the Museum. IEEE Transaction on Robotics and Automation. Workshop for Personal Robotics for Education, IEEE ICRA 2000.

Odorico, A. (2005). La robótica desde una perspectiva pedagógica. Revista de Informática Educativa y Medios Audiovisuales, 2(5), 33-48.

Odorico, A., Lage, F., y Cataldi, Z. (2009). Educación en robotica, una tecnología integradora. (Z. C. Fernando Lage, Ed.).

Ortíz, J., Rennola, L., y Johnny, B. (2005). Módulo educativo Multimedia para la enseñanza de dinámica y control de procesos. Acción pedagógica (14), 96-103.

Papert, S. (1995). La maquina de los niños. Buenos Aires: Paidos.

Payá, L., Reinoso, O., Gil, A., y Jiménez, L. (2007). Plataforma Distribuida para la Realización de Prácticas de Robótica Móvil a través de Internet. Información Tecnológica, Vol. 18(6), 27-38.

Pérez, M. (2004). Desarrollo de habilidades cognitivas en niños(as) orientado en la metacognición a través de la interacción con robots moviles en ambientes de realidad virtual. Bucaramanga.

Perteet, B., McClintock, J., y Fierro, R. (2007). A Multi-Vehicle Framework for the Development of Robotic Games: The Marco Polo Case. Robotics and Automation. 2007 IEEE International Conference., 3717-3722.

Piepmeier, J., Bishop, B., y Knowles, K. (2003 ). Modern robotics engineering instruction. Robotics \& Automation Magazine, IEEE, 10(2), 33-37.

Pinto, M., Barrera, N., y Pérez, W. (2010). Uso de la robótica educativa como herramienta en los procesos de enseñanza. Ingeniería, Investigación y Desarrollo $(I 2+D)$, 15-23.

Pittí, K., Curto, B., y Moreno, V. (2010). "Experiencias construccionistas con robótica educativa en el centro internacional de tecnologías avanzadas". Buenas prácticas de enseñanza con TIC.

Plestina, V., Turic, H., y Papic, V. (2007 ). Constructive education approach: robot soccer. Information Technology Interfaces, 2007. ITI 2007. 29th International Conference, 425-430. 
Raz, T. (1989). Graphics robot simulator for teaching introductory robotics Education. IEEE Transactions on, 32(2), 153-159.

Ruiz, N. (s.f.). Escuela del tercer milenio. Bogota: Prolibros.

Shuying, Zhao, et al. (2008). Research on Robotic Education Based on LEGO Bricks. Computer Science and Software Engineering, 2008 International Conference., 5, 733-736.

Sklar, E., Parsons, S., y Stone, P. (2004). Using RoboCup in University-Level Computer Science Education. ACM Journal on Educational Resources in Computin. 4(2).

Smith, C. (1997). This robot belongs to.....whom?. Robotics and Education, IEE. Colloquium, 91-92.

Tatsuya, Nomura, et al. (2004). Psychology in Human-Robot Communication: An Attempt through Investigation of Negative Attitudes and Anxiety toward Robots. Robot and Human Interactive Communication, 35-40.

Tec, B., Uc, J., Gonzalez, C., García, M., Escalante, M., y Montañez, T. (2010). Análisis Comparativo de dos Formas de Enseñar Matemáticas Básicas: Robots LEGO NXT y Animación con Scratch. In Proc. 7th IFAC Symp. Advances in Control Education. 40-47.

Turkle, S., y Papert, S. (1992). Epistemological Pluralism and the Revaluation of the Concrete. Journal of Mathematical Behavior, 11(1), 3-33.

Uldemolíns, J. (2008). Algunas reflexiones sobre la realidad del uso educativo de las TIC. Revista Iberoamericana de Educación., 5-25.

Vásquez, C. (2006). Aplicación del plc en robótica dentro de la educación superior como metodología de enseñanza. (Tesis Inedita de Pregrado), Universidad Mayor, Santiago de chile. Chile.

VEX Robotics [en línea]. (s.f.). Recuperado de http://www.vexrobotics.com/products/vex-robot-starterbundles

Virnes, M. (2008). Robotics in Special Needs Education. Proceeding of the 7th international conference on Interaction design and children. Session: Doctoral consortium., 29-32. ACM 978-1-59593-994-4

Weinberg, J. B., y Xudong, Y. (2003). Robotics in education: Low-cost platforms for teaching integrated systems. Robotics \& Automation Magazine, IEEE, 10(2), 4-6.

Xiaobo, T. (2006). An Autonomous Robotic Fish for Mobile Sensing. Proceedings of the 2006 IEEE/RSJ, International Conference on Intelligent Robots and Systems.

Xudong, Y., y Weinberg, J. (2003). Robotics in education: new platforms and environments. Robotics \& Automation Magazine. IEEE, 10(3), 3- 3. 